\title{
Développement et modèles de simulation de cultures
}

\author{
N Brisson, R Delécolle \\ INRA, station de bioclimatologie, BP 91 84143, Montfavet cedex, France
}

(reçu le 28 décembre 1990; accepté le 18 décembre 1991)

\begin{abstract}
Résumé - Dans un modèle de simulation de culture, le module de développement a un rôle pilote, car il tente de reproduire la logique stratégique de la plante. En s'appuyant sur des exemples de modélisation du développement chez le blé et le soja, on montre ici que les échelles choisies par les modélisateurs ne peuvent recouper entièrement celles qu'utilisent l'agronome ou le physiologiste. On envisage également les manières dont sont pris en compte les différents phénomènes qui sont sous la dépendance du schéma de développement (répartition, déterminisme du nombre d'organes reproducteurs, sénescence). On examine ensuite la forme générale des fonctions adoptées pour décrire l'action des facteurs du milieu sur le déroulement du schéma de développement, par l'intermédiaire de la notion de vitesse de développement. On aborde enfin les différentes méthodes d'estimation des coefficients génétiques qui permettent d'adapter le schéma de développement d'une espèce à ses divers génotypes.
\end{abstract}

développement / modèle de simulation / climat

Summary - Plant development in crop simulation models. This paper focusses on several aspects of the key role played by phasic development submodels within plant process models. Using examples of development models for wheat and soybean, it is shown that modellers cannot use developmental scales as used by agronomists or physiologists (pages 254-265, fig 1), and common ways to account for development-dependent phenomena in models (pages 256-257) (partitioning of assimilates, determination of final number of reproductive organs (figs 2 and 3), senescence) are considered. The general shape of functions used in models to describe the impact of environmental factors (pages 257-260) (temperature per se (fig 4) and through its effect on vernalisation status (fig 6), photoperiod (fig 5), water stress (fig 7)) on rates of development are examined. Some methods for estimating genetic parameters (pages 261-262) (from controlled (fig 8a) and field (fig 8b) condition experiments, through optimisation methods) are reviewed. 


\section{INTRODUCTION}

Les modèles mécanistes de simulation de culture sont des outils cognitifs qui tentent de reproduire analytiquement la dynamique de fonctionnement d'une plante ou d'une communauté de plantes avec un pas de temps défini.

Ils s'appuient sur 2 principes en interaction:

- un principe génétique manifesté par un programme préétabli qui fixe la durée des différentes phases du développement de la culture (végétative, reproductrice) en fonction des conditions de milieu et qui traduit une stratégie de l'espèce (ou de la variété) en termes de précocité, de pérennité ou d'adaptation au milieu. II s'agit là de la composante à long terme de l'évolution de cette culture, intégrée sur plusieurs pas de temps;

- un principe trophique, illustré par le dialogue source-puits (Warren Wilson, 1972; Gifford et Evans, 1981) qui caractérise les flux d'assimilats dans la plante entre des organes sources, actifs du point de vue photosynthétique, et des organes puits, consommateurs de photosynthétats. Ce principe représente l'économie à court terme, le fonctionnement instantané de l'objet étudié.

La structure des modèles de simulation est dès lors articulée autour de la représentation formelle de ces 2 types de mécanismes, et comporte très généralement:

- un module simulant la croissance nette du couvert végétal, c'est à dire la production de biomasse basée sur l'interception et la transformation par l'appareil assimilateur du rayonnement photosynthétiquement actif, et modulée par les processus de sénescence;

- un modèle de développement qui a 2 fonctions:

- simuler l'enchaînement du programme génétique pour reconstituer les étapes essentielles de la vie de la culture: germination, floraison, élaboration d'une graine viable, sénescence à long terme par exemple;

- piloter le module de croissance en organisant, tout au long du cycle, l'ouverture et la fermeture des puits, ainsi que leur force. II peut également agir sur les sources en contrôlant la mise en place de l'appareil photosynthétique (initiation des feuilles) et en actionnant les remobilisations des parties végétatives vers les organes de stockage.
Le rôle tout à fait stratégique de ce module le fait apparaître comme le centre de décision du modèle. On constate, par ailleurs, que les lois de fonctionnement du compartiment de développement ou ses lois d'interaction avec le module de croissance varient beaucoup, dans leurs principes, d'un modèle à l'autre. Cela résulte du manque de connaissances fondamentales dans ce domaine, qui induit des représentations empiriques de la réalité du fonctionnement.

Convaincus que les progrès des modèles de simulation de culture passent, en particulier, par une meilleure approche de la description du développement, nous proposons une analyse de l'approche et de la représentation du développement dans une optique de modélisation, en nous appuyant sur l'exemple de modèles de la littérature, simulant des cultures de blé et de soja essentiellement.

\section{L'ÉCHELLE DE DÉVELOPPEMENT DES MODĖLES}

\section{Deux échelles de référence: l'échelle physiologique et l'échelle agronomique}

En créant les échelles de développement, on a cherché à définir des repères dans le déroulement du cycle pour mieux comprendre le fonctionnement de la plante (cas du physiologiste), ou pour améliorer l'efficacité des techniques agricoles (cas de l'agronome).

D'un point de vue physiologique, le développement s'exprime en termes de signaux initiateurs, de discontinuité dans le programme (germination, induction florale) et de différenciation d'organes. L'échelle organogénétique de développement qui découle de cette approche s'inscrit parfaitement dans le principe génétique. Malheureusement ce type d'échelle est difficile à appliquer expérimentalement, autrement que par des tests biologiques destructifs et lourds à mettre en œuvre.

C'est pourquoi l'agronome, plus pragmatique, s'attache à caractériser le changement d'aspect de la culture, ce qui le conduit à une échelle phénoménologique, où la composante "visible à l'œil nu» du repère établi est privilégiée. De plus, se plaçant au niveau d'une communauté de plantes, l'agronome introduit également une composante statistique. 


\section{L'échelle de type modélisation}

L'échelle de développement du modélisateur doit répondre à des objectifs qui, d'une certaine façon, intègrent les approches précédentes.

Les repères organogénétiques permettent, non seulement de révéler la logique du programme génétique, mais aussi de caler le cycle par rapport aux risques climatiques, puisque la sensibilité au gel ou à la sécheresse, par exemple, est accentuée à certains stades. Parmi ces repères, certains peuvent ne jouer aucun rôle vis-à-vis du pilotage de la croissance. Ils sont utilisés uniquement en tant qu'étapes intermédiaires, marquant une modification des fonctions de réponse de la plante à l'environnement (changement de seuil de température, fin d'action de la durée du jour, par exemple), et permettent de caler dans le temps les stades ultérieurs.

Les repères agronomiques, en dehors de leur intérêt pour les interventions agricoles, sont utiles lorsque les résultats intermédiaires d'un modèle doivent être comparés à des observations phénoménologiques du couvert (diagnostic au champ, télédétection), en vue notamment de pratiquer des corrections en cours de simulation. Les modèles de développement ne tiennent cependant pas compte de la variabilité du couvert que nous avons évoquée précédemment: il pourrait s'agir d'une amélioration importante.

Dans le cadre de sa fonction de pilotage trophique, l'échelle de développement du modélisateur doit fournir des repères d'apparition et de disparition d'organes identifiables en termes de sources et de puits. C'est pourquoi l'approche paraît ici plus morphogénétique.

En termes d'ouverture de puits, le modélisateur exploite au mieux les repères classiques de l'échelle phénoménologique (fig 1). Toutefois, lorsqu'il s'agit d'une espèce à croissance indéterminée, telle que le soja, il est difficile de définir ces stades, initiateurs du flux d'assimilats vers les organes reproducteurs, de façon simultanée pour l'ensemble des structures de la plante. Le même problème apparaît chez l'ensemble des espèces pour la mise en place des structures végétatives (feuilles en particulier). Cela conduit le modélisateur à identifier et à gérer chaque groupe de structures de même âge (notion de cohorte) indépendamment (ARCWHEAT pour les feuilles du blé: Weir et al, 1984; SOYGRO pour les gousses du soja: Wilkerson et al, 1985; Brisson, 1989).

Les difficultés deviennent plus aiguës lorsqu'il s'agit de caractériser les stades "d'arrêt" qui permettent de jouer sur la durée des phases en simulant leur fin (arrêt d'apparition des feuilles, fin de croissance de l'épi végétatif chez le blé, fin de mise en place des gousses chez le soja, fin de remplissage des grains, maturité). Ces stades ne comportent pas obligatoirement d'équivalents dans les autres échelles.

En ce qui concerne la mise en place des sources, c'est à dire des organes capables de photosynthétiser, il faut distinguer 2 processus :

- l'apparition, en tant que manifestations visibles, des groupes de structures végétatives telles que les feuilles et les talles chez le blé ou les nœuds chez le soja, qui obéit à un plastochrone appelé phyllochrone dans le cas des feuilles et plus précisément phyllotherme lorsqu'il est indexé aux sommes de degrés $\times$ jours,

- l'accumulation de surface foliaire, qui est modélisée le plus souvent à partir de la croissance pondérale des feuilles et quelquefois à partir d'une somme de degrés $\times$ jours (cas des modèles CERES: Ritchie et Otter, 1984; Jones et Kiniry, 1986).

Les phyllochrones doivent nécessairement être pris en compte dans une modélisation fine du couvert (description feuille par feuille, ou cohorte par cohorte). Mais, représentant des rythmes d'apparition macroscopiques, ils ne sont pas liés directement aux rythmes d'initiation des organes, au sens où la logique du programme génétique les détermine, puisque l'apparition à l'œil des organes suppose une phase de croissance après l'initiation. Ces phyllochrones sont donc généralement des paramètres des modèles, déduits des conditions de milieu par une relation empirique.

Il semble donc difficile d'utiliser les échelles de développement agronomique et physiologique dans une optique de modélisation, et force est de constater que le modélisateur a besoin d'une échelle de développement adaptée à ses besoins, échelle qui doit faire l'objet d'une définition claire d'un point de vue expérimental, afin de procéder au calage correct des modèles. 


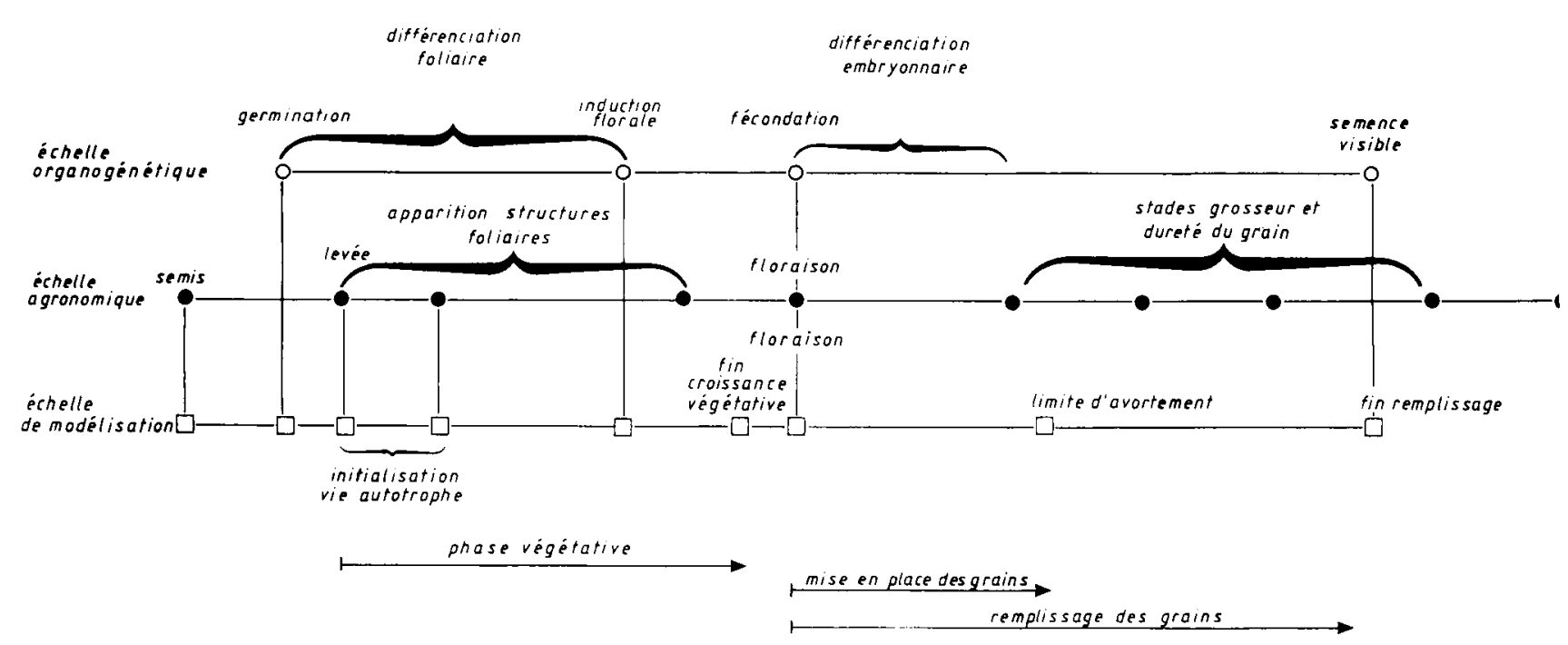

Fig 1. Différentes échelles de développement (cas d'une plante annuelle cultivée pour ses graines) : essai de représentation synthétique.

\section{PHÉNOMĖNES SOUS LA DÉPENDANCE DU SCHÉMA DE DÉVELOPPEMENT DANS LES MODĖLES}

\section{Gestion du schéma de répartition}

Dans les modèles de simulation de cultures, les assimilats formés à chaque pas de temps sont distribués aux différents organes suivant des coefficients de répartition dont la valeur varie selon les phases de développement, afin de tenir compte de l'apparition ou de l'extinction des puits. La formalisation de l'évolution de ces coefficients est intimement liée à la logique choisie pour modéliser l'échelle de développement.

Ainsi, dans les modèles relatifs à des espèces à croissance déterminée, les coefficients de répartition évoluent, soit suivant des fonctions en escalier, pour des échelles où le temps phénologique est discontinu (tableau de valeurs a priori pour ARCWHEATt, valeurs a priori ou fonctions instantanées des variables d'état dans CERES-Wheat, Richtie et Otter, 1984), soit suivant des fonctions continues pour des échelles qui le sont également (cas du modèle WHEAT: Van Keulen et Seligman, 1987, qui exprime l'état de développement de la culture dans une phase relativement à la durée totale de la phase).

Pour une espèce à croissance indéterminée, il est indispensable d'introduire en outre une notion de priorité: généralement les or-

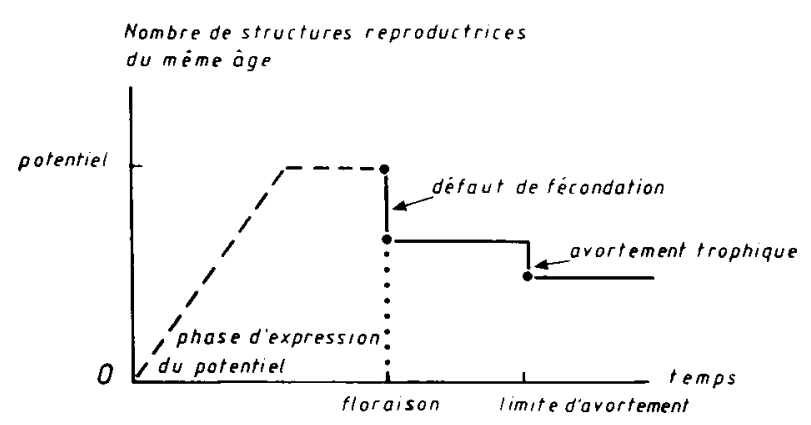

Fig 2. Représentation des phénomènes liés à la mise en place du nombre de structures reproductrices du même âge (cas général).

ganes reproducteurs (grains et enveloppes) sont prioritaires sur les parties végétatives. Cependant, cette allocation préférentielle doit être couplée avec une estimation de la taille maximale des puits (SOYGRO).

\section{Nombre d'organes reproducteurs}

Si l'on raisonne au niveau d'une cohorte de structures reproductrices, on peut définir des stades critiques pendant lesquels se situe le passage d'un nombre potentiel de structures (considéré comme le résultat d'interactions génotype - milieu), à un nombre réel (fig 2).

Aussi, pour estimer le nombre de structures, le modélisateur doit-il se donner les moyens de caractériser ce schéma avec le calcul des stades critiques, la définition des valeurs intermédiaires du nombre de structures ou la mise au point de lois de passage 
analytique ou empiriques. Le manque de connaissance dans ce domaine touchant au déterminisme du nombre d'organes et la vrajsemblable diversité de celui-ci (trophique, génétique, hormonal, environnemental ?) induit des approches variables en fonction de l'espèce et du modélisateur.

Ainsi utilise-t-on dans le modèle ARCWHEAT une relation empirique entre l'accumulation de matière sèche dans l'épi pendant la phase d'expression du potentiel et le nombre de grains. Le stade début de croissance de l'épi correspond au potentiel zéro, et la relation proposée intègre la phase potentielle ainsi que les processus de défaut de fécondation. En revanche, cette approche néglige l'avortement trophique, ce qui permet de ne pas simuler le stade limite d'avortement.

Dans le modèle SOYGRO, la nécessité de définir le nombre d'organes pour simuler la taille des puits, utile au schéma de répartition, conduit à une démarche plus analytique. Le nombre d'organes potentiels est donné comme un paramètre génétique, ce qui permet de na pas modéliser la phase potentielle. Le défaut de fécondation est simulé comme la conjugaison d'un facteur photothermique et d'un facteur trophique, tandis que l'avortement trophique est relié à l'adéquation entre l'offre journalière en assimilats et la vitesse de croissance potentielle des grains. De plus, le stade limite d'avortement est estimé de façon très empirique comme se situant 10 jours après la floraison.

Ce schéma, applicable à une cohorte de gousses du même âge, se répète pendant toute la durée de la floraison, d'où la définition, chez le soja, d'une réelle phase de mise en place des structures (voir fig 3). Chez le blé, cette phase est réduite à un stade.

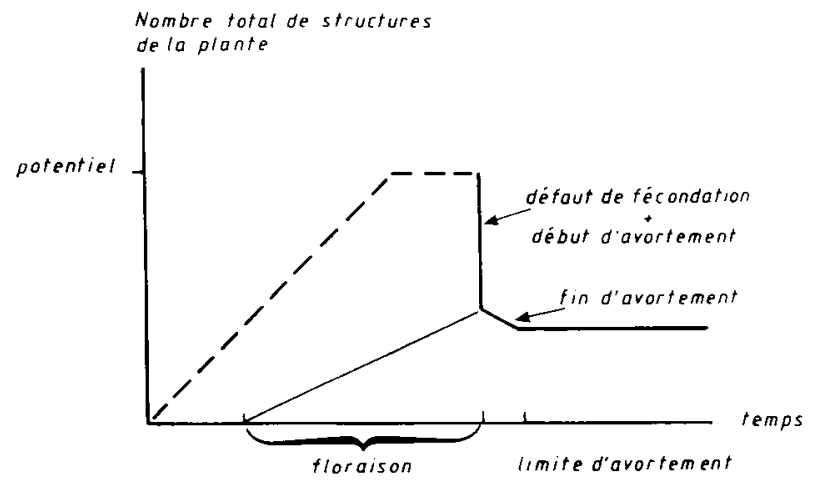

Fig 3. Représentation de la mise en place du nombre total de structures reproductrices de la plante pour une espèce à croissance indéterminée (exemple du soja).

\section{Sénescence}

La variation des durées de vie observée pour des feuilles de différentes espèces (Dale et Milthorpe, 1983) indique que les processus de sénescence dépendent du programme génétique de la plante. L'explication la plus couramment invoquée est que le passage de la plante à la vie reproductrice induit une compétition trophique entre les organes végétatifs et les organes reproducteurs. Cependant, des expérimentations (citées par Salisbury et Ross, 1985) ont démontré l'intervention de facteurs hormonaux qui auraient pour fonction de détourner, au niveau du phloème, les assimilats vers les grains. II semble donc que les processus de sénescence résultent également d'étroites relations entre croissance et développement, que l'on peut définir comme une rupture d'équilibre entre les dégradations des protéines de la photosynthèse et leur biosynthèse, cette rupture étant pilotée par le calendrier de la plante, via un mécanisme hormonal.

Par conséquent, la sénescence correspond à une perte d'activité qui se traduit, en termes de modélisation, par une perte de matière sèche pour l'organe, et qui débute dès que la plante est autotrophe. En général, la vitesse de sénescence de la matière sèche augmente avec l'âge de la plante et les contraintes hydriques et minérales qu'elle subit. À cette perte nette pour la plante, il faut ajouter les remobilisations, de nature protéique ou glucidique, vers les organes de stockage qui interviennent pendant la phase de remplissage. La vitesse de développement peut déterminer la vitesse avec laquelle se produisent ces remobilisations.

\section{FORMALISATION DES PHÉNOMĖNES DE DÉVELOPPEMENT}

Les modèles de développement servent donc à simuler le déclenchement des stades qui permettent de représenter la logique phasique de la plante. Ils fournissent, pour ce faire, une échelle continue, sorte d'horloge physiologique, qui peut être appliquée comme une base de temps à l'évolution des processus physiologiques (Delécolle, 1987). Les facteurs explicatifs du développement sont généralement la 
température et la photopériode. II arrive également que l'on introduise le stress hydrique.

\section{Notions de parcours et de vitesse de développement}

La formalisation du développement s'appuie sur la notion de parcours de développement: on considère en effet que la réalisation d'une phase de développement comprise entre 2 stades $S_{1}$ et $S_{2}$ est équivalente à un parcours effectué par la plante entre ces 2 stades (Durand, 1967). Cette équivalence avec une distance permet de définir la longueur $L$ d'une phase de développement (mesurée en unités arbitraires, à ne pas confondre avec la durée qui est mesurée en unités de temps). La vitesse de développement $V$ se définit de facto par rapport au temps sur un élément infinitésimal de parcours de développement $d l$ :

$$
V=\frac{\mathrm{d} l}{\mathrm{~d} t},
$$

$V$ variant au cours du temps en fonction des variables du milieu. L'on a:

$$
L=\int_{S_{2}}^{S_{1}} \mathrm{~d} /=\int_{t\left(S_{1}\right)}^{t\left(S_{2}\right)} V(t) \mathrm{d} t
$$

Si l'on suppose par exemple que $V$ varie linéairement avec la température $T$, suivant une loi $V=a\left(T-T_{0}\right)$, on aura:

$$
L=\int_{t\left(S_{1}\right)}^{t\left(S_{2}\right)} a\left[T(t)-T_{0}\right] d t
$$

De façon pratique, on utilise pour $T(t)$ les températures moyennes sur une unité de temps (généralement 1 jour), $T M_{j}$ :

$T M_{j}=\frac{\int_{j} T(t) \mathrm{d} t}{\int_{j} \mathrm{~d} t} \operatorname{avec} \int_{j} \mathrm{~d} t=1$ unité (1 jour),

et on donne une formulation directe du parcours de développement:

$$
\begin{array}{ll}
L=a \sum\left(T M_{j}-T_{0}\right) \times 1 & \text { (1 représentant } \\
\text { l'unité de temps). }
\end{array}
$$

De fait, le parcours de développement n'est pas réversible, aussi impose-t-on la condition supplémentaire $T M_{j}-T_{0}=0$ si $T M_{j}<T_{0}$.

On constate que, sous l'hypothèse de dépendance linéaire de la vitesse de développement à la température, le parcours de développement est homogène en unités au produit d'une température par un temps, ce qui justifie la mesure de ce parcours en ${ }^{\circ} \mathrm{C} \times \mathrm{j}$ (ou, par usage, en somme de ${ }^{\circ} \mathrm{C} \times \mathrm{j}$ ). Ce formalisme permet de graduer l'échelle de développement en somme de la variable climatique, d'où le terme sommes de températures (les expressions anglaises thermal time et progress towards stage $S$ paraissant d'ailleurs mieux adaptées).

II convient ici de différencier la relation réelle (modèle théorique) qui relie la vitesse de développement aux facteurs du milieu et que l'on peut décrire par des expériences en conditions contrôlées, de la relation utilisée dans la modélisation (modèle empirique). Si le modèle théorique est raisonnablement proche de la linéarité dans le cas de l'influence de la photopériode, à condition d'admettre un phénomène à seuil (Hunt, 1979; Major, 1980), il n'en va pas de même pour la température. De nombreux résultats montrent en effet que la linéarité de la réponse n'est acceptable que pour une partie de la gamme des températures non létales (Angus et al, 1981; Garcia-Huidobro et al, 1982). Le formalisme des sommes de ${ }^{\circ} \mathrm{C} \times \mathrm{j}$ présente donc 2 inconvénients: la variation, selon les phases, des seuils apparents de température $T_{0}$ estimés et la mauvaise estimation de la vitesse hors de la gamme de températures où la réponse est véritablement linéaire (Porter et Delécolle, 1988).

Dans les cas où l'hypothèse de liaison linéaire ne peut être admise, un raisonnement analogue peut être appliqué au calcul de $L$. On obtiendra alors de $L$ une valeur comptée en unités phénologiques arbitraires. En fait, on se ramène généralement dans ce cas à une représentation en segments de droite de la courbe $V=f(T)$ ( $c f$ la courbe de vitesse de développement du soja utilisée par Jones et al, 1991).

\section{Courbes de réponses}

Delécolle et Guérif (1986) ont analysé la diversité des courbes de réponse proposées dans la littérature, en fonction des espèces et des phases considérées. En effet, les facteurs du milieu agissent différemment d'une phase à l'autre, et la modélisation de la succession de ces phases devient rapidement complexe.

Toutefois, on observe que les différentes démarches adoptées dans les modèles de dé- 
veloppement reviennent à exprimer la vitesse instantanée comme le produit de plusieurs facteurs :

$$
V_{i}=\theta_{i}(T) \times \varphi_{i}(P) \times v_{i}(T) \times \psi_{i}(D)
$$

où : $\theta=$ effet accélérateur des températures; $\varphi=$ effet photopériodique; $v=$ effet vernalisant des températures; $\psi=$ effet du stress hydrique; $i=$ indice de la phase; $T=$ température; $P=$ photopériode; $D=$ déficit hydrique (exprimé en fonction de la teneur en eau du sol ou du potentiel hydrique foliaire ou de la température de surface du couvert, etc)

Les fonctions $\theta, \varphi, v$ et $\psi$ peuvent donc varier d'une phase à l'autre, ou même ne pas intervenir (cas particulier où elles sont égales à 1). On considère très souvent l'effet thermique, $\theta_{i}$, comme le moteur des processus de développement, les autres effets ne jouant qu'un rôle modulateur de l'action de la température. En conséquence, la somme des $V_{1}$ élémentaires pendant la phase $i$ a la dimension de la somme des $\theta_{i}$. Comme on l'a vu au paragraphe précédent, cette somme est généralement exprimée en ${ }^{\circ} \mathrm{C} \times \mathrm{j}$. Les autres facteurs peuvent donc être modélisés comme des fonctions de réduction sans unité variant entre 0 et 1 (sauf cas du déficit hydrique qui peut induire une accélération de la vitesse de développement et donc conduire à des valeurs supérieures à 1 ). En réalité, il peut exister des interactions entre le facteur thermique et les facteurs photopériodique ou hydrique. Dans ce cas, les fonctions $\varphi$ et $\psi$ dépendent aussi de la température.

Quelle que soit leur forme, ces fonctions font intervenir un nombre non réductible de paramètres. Voici des courbes de forme simple qui, cependant, respectent la dynamique d'action de chaque facteur:

\section{Effet de la température :}

La fig 4 montre une approximation classique de la réponse réelle (continue) du développement à la température (Soygro, Ceres), tenant compte de l'existence de températures cardinales propres à chaque végétal: $T_{\operatorname{mini}}$ (aussi appelée température de base ou zéro de végétation) et $T_{\operatorname{maxi}}$ définissent l'intervalle en-dehors duquel la vitesse de développement est apparemment nulle, $T_{\text {opt }}$ est la valeur qui maximise cette vitesse. On trouve aussi fréquemment des courbes ne faisant pas intervenir un effet négatif des fortes températures.

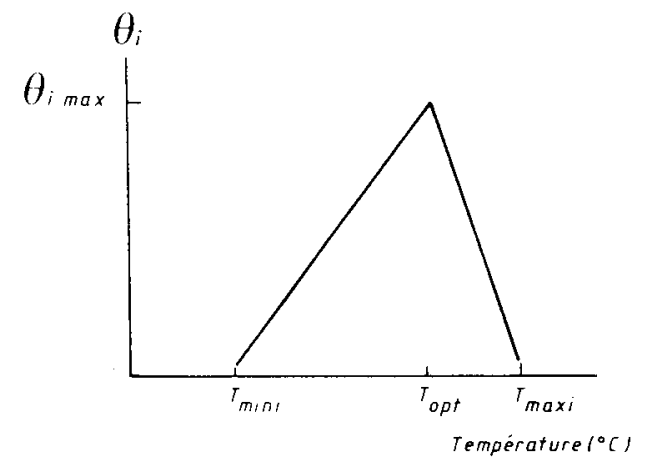

Fig 4. Courbe de réponse type de la vitesse de développement à la température ( $T_{\operatorname{mini}}$, $T_{\text {opt }}$ et $T_{\text {maxi }}$ étant des températures cardinales).

\section{Effet de la photopériode:}

La fonction de photopériode $\varphi_{i}$ varie selon le type de plante: plante de jours courts ou plante de jours longs. La fig 5 propose une action discontinue de ce facteur, où la vitesse de développement n'est affectée qu'entre les seuils photopériodiques $P_{0}$ et $P_{S}$ (Major, 1980). À l'extérieur de cet intervalle, la vitesse de développement est, soit minimale $\left(1 / G_{\operatorname{maxi}}\right.$ avec $G_{\operatorname{maxi}}=$ longueur maximale de la phase) soit maximale $\left(1 / G_{\operatorname{mini}}\right.$ avec $G_{\min }=$ longueur minimale de la phase). Après normalisation, $\varphi_{i}$ varie entre $G_{\operatorname{mini}} / G_{\operatorname{maxi}}$ et. 1 . Si $G_{\max i}$ tend vers l'infini (le rapport $G_{\min i} / G_{\max i}$ tend vers 0 ) alors l'effet photopériodique peut induire une annulation de la vitesse de développement (exemple de la betterave). De plus, si l'on met en évidence une interaction entre effet thermique et effet photopériodique, une façon simple de modéliser cette interaction est d'exprimer les longueurs des phases $\left(G_{\text {mini }} / G_{\text {maxi }}\right)$ non pas en unités de temps mais en unités phénologiques (sommes de degrés $\times$ jours par exemple, cas de Soygro).

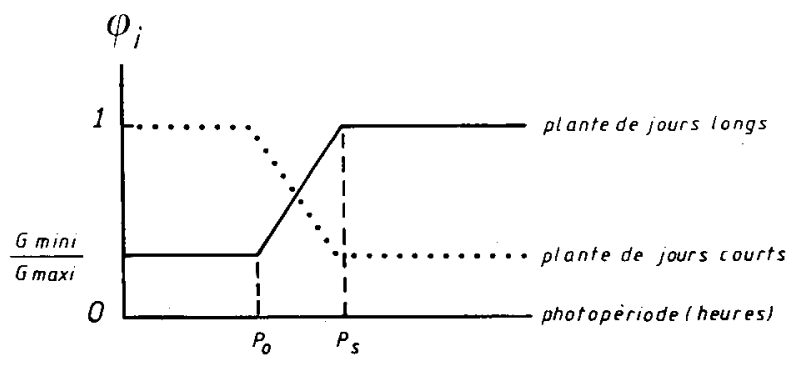

Fig 5. Courbe type de pondération de la vitesse de développement par la photopériode. 


\section{Effet vernalisant :}

La vernalisation est sans doute le facteur le plus délicat à modéliser. Dans ce cas en effet, la vitesse de développement n'est pas influencée directement par la température, mais par "l'état de vernalisation» de la plante. Celui-ci est donc en lui-même l'intégration sur le temps d'une réponse élémentaire. La fig 6 présente la forme généralement admise (Maas et Arkin 1980; Ritchie et Otter 1984; Weir et al, 1984; Reinink et al, 1986) de cette réponse élémentaire, $R_{v}$, exprimée en fraction de jours vernalisants, et qui nécessite la définition de plusieurs températures cardinales (4 dans notre cas). L'état de vernalisation à l'instant $t_{j}$ à partir d'un stade $S_{i}$ est dès lors égal à :

$$
\sum_{t\left(S_{j}\right)}^{t_{j}} R_{v}
$$

et $v_{\text {; }}$ peut être défini comme le rapport de cette grandeur au nombre total de jours nécessaire à atteindre la pleine vernalisation en conditions optimales (températures comprises entre $T_{\text {opt } 1}$ et $\left.T_{\text {opt2 }}\right)$. Weir et al (1984) utilisent pour le blé d'hiver une expression normée de $v_{i}$ afin de prendre en compte une durée minimale $V_{0}$ d'exposition aux températures vernalisantes:

$$
v_{i}=\frac{\sum R_{V}-V_{0}}{V_{\text {sat }}-V_{0}}\left(V_{0}>0\right)
$$

Ritchie (1991) propose une formulation qui s'adapte également aux blés à vernalisation facultative :

$$
v_{i}=1-k \times\left(50-\sum R_{v}\right),
$$

où 50 est le nombre de jours vernalisants nécessaires aux blés les plus exigeants, et $k$ est un coefficient de sensibilité variétale. On peut voir que si $V_{\text {sat }}=50$, si $k=\left(V_{\text {sat }}-V_{0}\right)^{-1}$ et si $V_{0}$ n'est pas borné à 0 , les 2 expressions sont équivalentes.

\section{Effet du stress hydrique :}

Les effets du stress hydrique sur le développement sont assez mal connus. Les stress légers auraient tendance à accélérer le cycle en réponse à l'élévation de température du végétal. Par contre, un stress sévère provoquerait le ralentissement, voire l'arrêt du développement (Bradford et Hsiao, 1982; Evans,

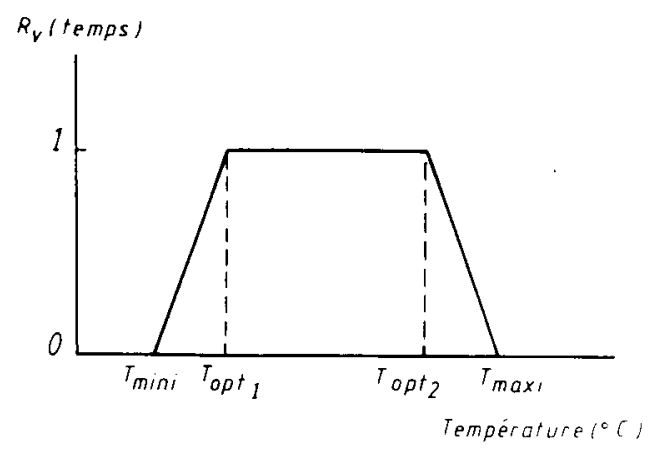

Fig 6. Courbe type de calcul de la participation de chaque unité de temps à l'état de vernalisation de la plante (basée sur des températures cardinales $T_{\text {mini, }} T_{\text {opt1 }}$ et $T_{\text {opt } 2 \text {, la }}$ courbe définit chaque unité de temps comme une fraction $R v$ d'unité de temps vernalisante, qui est cumulée pour estimer l'état de vernalisation de la plante. La vernalisation est réalisée quand l'état de vernalisation est égal à 1).

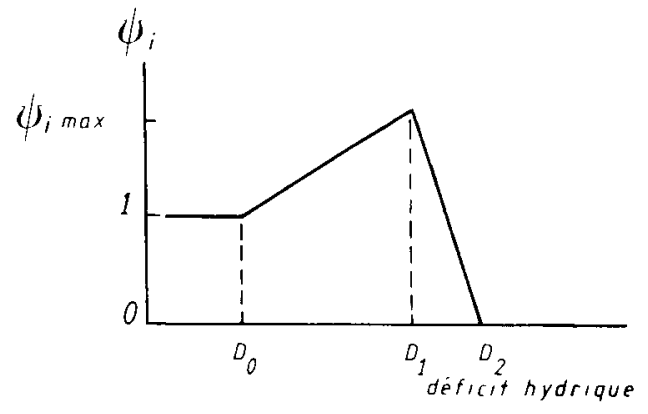

Fig 7. Proposition de courbe de pondération de la vitesse de développement par le déficit hydrique.

1984; Hodges et French, 1985; Van Keulen et Seligman, 1987), mais ces effets sont très rarement pris en compte dans les modèles (effet frein pour la phase de mise en place du nombre de gousses dans Soygro). On pourrait cependant suggérer une courbe de réponse du type de celle de la figure 7 où $D_{0}$, $D_{1}$ et $D_{2}$ sont 3 valeurs cardinales du déficit hydrique $(D)$ : en dessous d'un seuil optimal de déficit hydrique, $D_{0}$, la vitesse de développement n'est pas affectée; entre $D_{0}$ et le seuil $D_{1}$, elle est accélérée puis ralentie entre $D_{1}$ et $D_{2}$ pour s'annuler après $D_{2}$. Le déficit hydrique peut être exprimé en fonction de la teneur en eau du sol ou du potentiel hydrique de la plante ou encore de la température de surface du couvert végétal, ce qui nécessite l'utilisation annexe d'un modèle de bilan hydrique. La fonction $\psi_{i}$ s'exprime alors comme une vitesse de développement normalisée par la vitesse optimale correspondant à $D<D_{0}$. 


\section{ESTIMATION DES PARAMĖTRES GÉNÉTIQUES}

Ces différentes fonctions de réponse s'adaptent aux différentes sensibilités spécifiques et variétales grâce aux valeurs cardinales et à d'autres paramètres (nombre total de jours vernalisants nécessaires, ou $\theta_{\text {imax }}$, par exemple). Ces valeurs sont généralement appelées paramètres génétiques. L'un des principaux problèmes posés par la modélisation du développement est l'estimation des valeurs de ces paramètres pour une espèce et une variété données.

Cette estimation est généralement réalisée à partir de résultats obtenus en conditions contrôlées, pour chaque facteur climatique pris individuellement et pour un nombre réduit de variétés, à cause du coût induit par de telles expérimentations. Dans le cas de l'effet de la température, par exemple, cette approche consistera à placer une variété dans une gamme de conditions thermiques (généralement constantes) différentes, à repérer pour chacune d'elles la durée $d$ de réalisation de la phase examinée, transformer cette durée en vitesse de développement, $1 / d$, et à ajuster par une fonction $f(T, \mu)$ les points observés, $\mu$ représentant le jeu de paramètres nécessaire à la description de $t$. L'ajustement fournit une fonction $f(T, \hat{\mu}) \hat{\mu}$ représentant les valeurs estimées des paramètres (fig $8 \mathrm{a}$ ).

On peut également tenter cette estimation à partir de données obtenues en conditions naturelles, en utilisant des séries de données (semis décalés, expérimentations pluriannuelles). II est alors possible de procéder comme dans le cas des conditions contrôlées, mais en utilisant comme variables explicatives d'un modèle simple les valeurs moyennes des variables climatiques observées sur la phase phénologique considérée (Porter et al, 1987).

Dans notre exemple de l'effet de la température, on représentera la vitesse de développement comme l'inverse de la durée calendaire de réalisation du stade étudié, et $\mathrm{ce}$, en fonction de la température moyenne pendant cette phase (fig 8b), fournissant ainsi une réponse ajustée de type $1 / d=\hat{a}\left(T-T_{0}\right)$. Cette méthode fournit en général des résultats bruités (Baker et Gallagher, 1983), ce qui la rend difficilement utilisable lorsqu'on travaille sur plus de 2 variables climatiques.

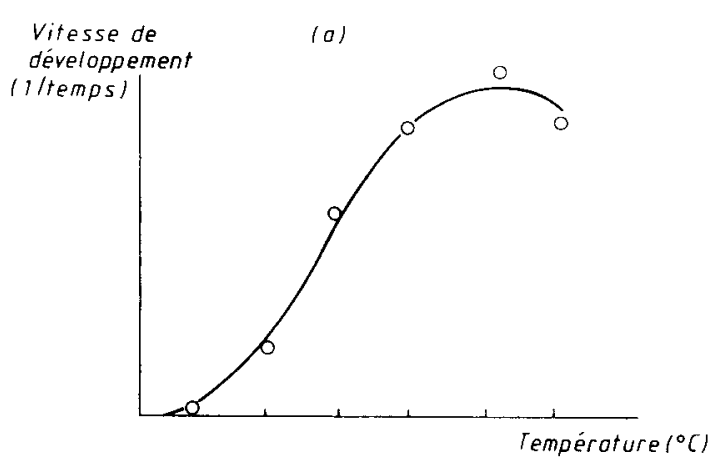

(b)

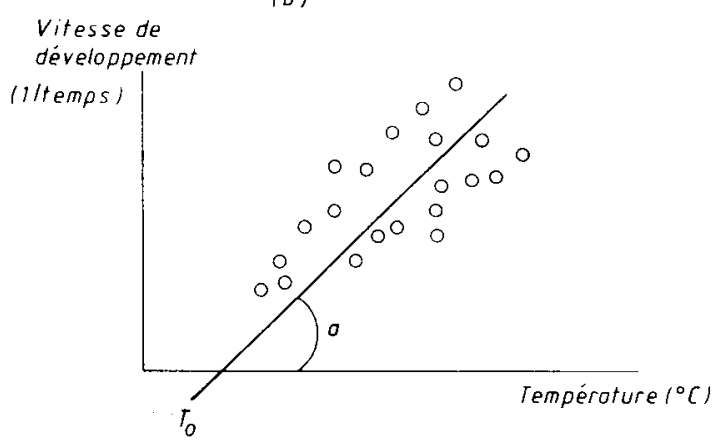

Fig 8. Exemples d'ajustement de la réponse de la vitesse de développement à la température a. à partir d'observations en conditions thermiques contrôlées; b. à partir d'observations obtenues en conditions naturelles (l'abscisse de chaque point est la température moyenne observée sur la phase dont on mesure la vitesse de réalisation); $O$ : points expérimentaux.

Dans le cas de la recherche d'une loi complexe, on peut également procéder par minimisation: partant d'une série d'observations de dates, $S$, du stade étudié, on recherchera, en reprenant ici notre exemple du paragraphe Courbes de réponse (pages 258259), les valeurs des paramètres $\mu$ de la fonction $V_{i}$ :

$$
V_{i}=\theta_{i}\left(T, \mu_{1}\right) \times \varphi_{i}\left(P, \mu_{2}\right) \times v_{1}\left(T, \mu_{3}\right) \times \psi_{1}\left(D, \mu_{4}\right)
$$

qui minimisent la distance globale entre ces dates, $S$, observées et les dates $S^{*}$ simulées par le modèle $V_{i}$ muni des paramètres $\mu^{*}$. Pour chaque observation, $S^{*}$ est obtenu comme la date qui réalise la condition:

$$
\begin{array}{r}
\sum_{i=1}^{s *} V_{i}=\theta_{i}\left(T, \mu_{1}^{*}\right) \times \varphi_{i}\left(P, \mu_{2}^{*}\right) \times v_{i}\left(T, \mu_{3}^{*}\right) \\
\times \Psi_{i}\left(D, \mu_{4}^{*}\right)=1
\end{array}
$$

et sur toute la série, on minimise un critère d'adéquation, généralement $\Sigma\left(S-S^{*}\right)^{2}$ minimum (Hammer et al, 1989). Cette méthode est théoriquement meilleure. II semble cependant qu'elle demande de nombreuses répéti- 
tions (observations) pour fournir des résultats stables.

\section{CONCLUSION}

La formalisation du développement joue un rôle clé dans les modèles de simulation des cultures. La nécessité dans ces modèles de se rapprocher de la réalité physiologique du fonctionnement de la plante (approche mécaniste) implique que les processus de développement utilisés soient cohérents avec cette approche. Or, les échelles de développement opérationnelles (celles de l'homme de terrain), qui s'appuient sur des critères de modification de la structure du couvert végétal, ne sont pas généralement compatibles avec les échelles de la modélisation. Cela peut présenter des inconvénients dans le cas où l'on veut utiliser de manière opé. rationnelle les modèles de simulation de culture, et être capable de vérifier, à partir de critères d'état du couvert (observables facilement), que les simulations sont phénologiquement synchrones à la réalité en cours de cycle (cas du couplage modèles mécanistes-télédétection: Maas, 1988).

La forme des fonctions de réponse du développement aux facteurs du milieu reste également très simpliste dans les modèles, ce qui pose problème pour simuler la réponse de la culture aux conditions climatiques extrêmes (dans la gamme de réponses non linéaire). Le coût et la complexité des études agrophysiologiques nécessaires à leur amélioration sont certainement en cause, mais des approches théoriques plus poussées, des recherches de descripteurs mathématiques plus adéquats (processus) seraient certainement nécessaires et permettraient de tenir compte des réponses non linéaires.

\section{REMERCIEMENTS}

Les auteurs expriment leur reconnaissance à R Bonhomme pour les nombreuses améliorations qu'il leur a suggérées.

\section{RÉFÉRENCES}

Angus JF, Cunningham RB, Moncur MW, McKenzie DH (1980) Phasic development in field crops. I Thermal response in the seedling phase. Field Crops Res 3, 365-368

Baker CK, Gallagher JN (1983) The development of winter wheat in the field. 2. The control of primordium initiation rate by temperature and photoperiod. J Agric Sci Camb 101, 337-344

Bradford KJ, Hsiao TC (1982) Physiological responses to moderate water stress. In: Phy siological Plant Ecology II, Springer Verlag, Berlin. New Series, 12B chap 9, 263-324

Brisson N (1989) Modèle de simulation de la culture du soja et de son fonctionnement hydrique. Estimation agrométéorologique des potentialités de production. Thèse INA-PG, $90 p$

Dale JE, Milthorpe FL (1983) The growth and functioning of leaves. (Dale JE et Milthorpe FL eds) Cambridge Univ Press, Cambridge, $151 p$

Delécolle R (1987) Cereal crop development models. In: Models in agriculture and forest re search (Miglietta F, ed) Proc IPRA workshop, San Miniato, 1-3 juin 1987, 107-111

Delécolle R, Guérif $M$ (1986) Les modèles agrométéorologiques de développement. Note in terne $n^{\circ} M 86 / 9$, station de bioclimatologie, INRA, Montfavet. Projet ESA “Utilité SAR», 40, $61 \mathrm{p}$

Durand R (1967) Action de la température et du rayonnement sur la croissance. Ann Physiol Vég 9, 5-27

Evans ML (1984) Functions of hormones at the cellular level of organisation. In: Encyclopedia of plant physiology New Series, Vol 10: Hormonal regulation of development (Scott TK ed) Springler Verlag, Berlin, 23-79

Garcia Huidobro J, Monteith JL, Squire GR (1982) Time, temperature and germination of pearl millet (Pennisetum typhoides $\mathrm{S}$ et $\mathrm{H}$ ). I. constant temperature. J Exp Bot 33 (133) : 288-296

Gifford RM, Evans LT (1981) Photosynthesis, carbon partitioning and yield. Ann Rev Plant Physiol 32, 485-509

Hammer GL, Vanderlip RL, Gibson G, Wade LJ, Henzell RG, Younger DR, Warren J, Dale AB (1989) Genotype-by-environment interaction in grain sorghum. II. Effects of temperature and photoperiod on ontogeny. Crop Sci, 29 : 376-384

Hodges T, French V (1985) SOYPHEN : soybean growth stages modeled from temperature, daylength and water availability. Agron $J 77,500$ 505 
Hunt LH (1979) Photoperiodic responses of winter wheats from different climatic regions. $Z$ Pflantz $82,70-80$

Jones CA, Kiniry-JR (1986) CERES - Maize, a simulation model of maize growth and development (Jones, Kiniry eds) Texas A \& M Univ Press, $194 p$

Jones WJ, Boote KJ, Jagtap SS, Mishoe JW (1991) Soybean development. In: Modeling Plant and Soil Systems (J Hanks and JT Ritchie eds) Agronomy Monograph no 31, ASA-CSSASSSA, 71-90

Keulen H (van), Seligman NG (1987) Simulation of water use, nitrogen nutrition and growth of a spring wheat crop. Simulation Monograph, Pudoc, Wageningen, $310 p$

Maas S (1988) Using satellite data to improve model estimates of crop yield. Agron J 80, 655-662

Maas SJ, Arkin GF (1980) TAMW : a wheat growth and development simulation model. Tex Agric Exp St, Blackland Research Center, Temple, Texas. Doc no 80-3, $124 \mathrm{p}$

Major DJ (1980) Photoperiod response characteristics controlling flowering of nine crops. Can $J$ Plant Sci 60, 777-784

Porter JR et 19 collaborateurs (1987) An analysis of morphological development stages in Avalon winter wheat crops with different sowing dates and at ten sites in England and Scotland. $J$ Agric Sci, Camb 109: 107-121
Porter JR, Delécolle R (1988) Interaction of temperature with other environmental factors in controlling the development of plants. In: Plants and Temperature, Symp Soc Exp Biol (SP Long and FI Woodward eds) 42 : 133-156

Reinink K, Jorritsma I, Darwinkel A (1986) Adaptation of the AFRC wheat phenology model for Dutch conditions. Neth J Agric Sci, 34 1-13

Ritchie JT (1991) Wheat phasic development. In: Modeling Plant and Soil Systems (J Hanks and JT Ritchie eds) Agronomy Monograph no 31 , ASA-CSSA-SSSA : $31-54$

Ritchie JT, Otter S (1984) Ceres-Wheat: a useroriented wheat yield model. Preliminary documentation, Agristars Publication No YM-U3-04442-JSC-18892

Salisbury EB, Ross CW (1985) Plant physiology. Third edition. Wadsworth publishing company, $540 p$

Warren-Wilson J (1972) Control of crop processes in controlled environment (AR Rees, KE Cockshull, DW Hand, RG Hurd eds) Acad Press London, 7-30

Weir AH, Bragg PL, Porter JR, Rayner JH (1984) A winter wheat crop simulation model without water or nutrient limitations. J Agric Sci Camb, $102,371-382$

Wilkerson GG, Jones JW, Boote KJ, Mishoe JW (1985) SOYGRO V5.0: Soybean crop growth and yield model. Int Rep Gainesville Univ Florida USA, $220 p$ 